\title{
INFLUENCE DU SUBSTRAT ET DU GENOTYPE SUR LA CROISSANCE ET LA SURVIE AU COURS DE LA RÉSORPTION VITELLINE CHEZ LA TRUITE COMMUNE (SALMO TRUTTA L.)
}

\author{
Francine KRIEG (1), R. GUYOMARD (1), G. MAISSE (2) et B. CHEVASSUS (1)
}

(1) Laboratoire de Génétique des Poissons, INRA/CRJ. 78350 - JOUY-EN-JOSAS.

(2) Laboratoire de Physiologie des Poissons, INRA, Campus de Beaulieu, 35000 - RENNES.

Reçu le 16 avril 1988

Accepté le 27 decembre 1988

Aeceived 16 April, 1988

Accepted 27 December, 1988

\section{RESUME}

Nous avcns étudié les performances de survie et de croissance, au cours de la résorption vitelline, de descendants issus dune souche domestique, d'une souche sauvage et des deux croisements réciproques entre celles-ci. Les différents génotypes ont été élevés sur substrats lisses et rugueux. Nous avons pu mettre en évidence un effet positif du substrat rugueux sur la croissance et la survie de tous les lots ainsi qu'un effet souche en faveur de la souche domestique. Par contre, aucun effet génétique significatif n’a pu être décelé.

\section{SUBSTRATE AND GENOTYPE EFFECT ON THE SURVIVAL AND GROWTH PERFORMANCES DURING YOLK RESORPTION IN BAOWN TROUT (SALMO TRUTTA L.)}

\section{SUMMARY}

Survival and growth performances during yolk resorption have been studied on a domesticated and a wild strains as well as on crossbreds between these two strains. These genotypes were hatched on a flat and an artificial substrates. Positive effects of the artificial substrate and the domesticated strain on survival and growth rates were demonstrated. However, no significant genetic effect was detected.

\section{INTRODUCTION}

L'utilisation, lors de la résorption vitelline, d'un substrat rugueux imitant les conditions naturelles est maintenant une technique bien connue pour l'élevage des salmonidés, notamment du saumon atlantique (Salmo salar) et de la truite commune (Salmo trutta). Un gain de poids en fin de résorption et parfois une amélioration de la survie sont ainsi obtenus (LEON, 1975; GAIGNON et PROUZET, 1982; HANSEN et TORRISSEN, 1984; HANSEN et MOLLER, 1985; ERIKSSON et WESTLUND, 1985 sur le saumon atlantique; ERIKSSON et WESTLUND, 1983; HANSEN, 1985 sur la truite de mer).

Chez la truite commune, l'élevage de souches sauvages destinées au repeuplement est souvent considéré comme difficile. Ainsi MAISSE et al. (1983) ont montré que le croisement de femelles domestiques (souche de la Pisciculture Fédérale de la Gouarnais) avec des mâles sauvages capturés en rivière fournissait des alevins présentant en pisciculture une croissance et un taux de survie plus faibles que ceux obtenus en utilisant des máles domestiques. De méme, nous avons pu montrer en comparant la méme souche domestique aux descendants de géniteurs sauvages capturrés sur l'Elorn (GUYOMARD et CHEVASSUS, 1985) que ceux-ci avaient une croissance environ deux fois plus lente et un taux de survie très inférieur (25\% du témoin). En outre, les descendants des croisements entre ces deux souches présentaient des résultats intermédiaires, ce qui permettait d'interpréter cette différence comme étant essentiellement d'origine génétique.

Des études génétiques utilisant d'autres méthodes (polymorphisme protéique) ont montré que la population de l'Elorn se différencie nettement de toutes les populations de pisciculture utilisées dans cette région pour le repeuplement (KRIEG et GUYOMARD, 1985) et constitue donc bien une population naturelle non domestiquée antérieurement.

Nous avons cherché à préciser si cette différence entre ces deux souches apparaissait précocément avant le début d'alimentation, et si l'utilisation d'un substrat rugueux pendant la résorption de la vésicule vitelline pouvait modifier cette différence et améliorer les performances de la souche "Elorn". 


\section{MATÉRIEL ET MÉTHODES}

\section{MATÉRIEL ANIMAL}

Deux groupes de reproducteurs ont été utilisés :

- des géniteurs des deux souches élevés à la pisciculture INRA-CSP de Gournay sur Aronde (Oise) et introduits dans cette pisciculture en 1983 au stade d'œufs embryonnés. A partir de ces reproducteurs, un croisement diallèle a été réalisé pour produire les quatre génotypes suivants: E X E (4 femelles Elorn $\times 6$ mâles Elorn), E x G (4 femelles Elorn $\times 6$ mâles Gouarnais), $G \times E$ (4 femelles Gouarnais $\times 6$ mâles Elorn), $G \times G$ ( 4 femelles Gouarnais $\times 4$ mã les Gouarnais). Ces lots ont été incubés à $10^{\circ} \pm 0,5^{\circ} \mathrm{C}$ dans les installations recyclées de l'INRA à Jouy-en-Josas.

- Des géniteurs (15 femelles et 15 måles) prélevés à nouveau sur l'Elorn en 1986, afin de juger d'un éventuel effet de l'élevage des reproducteurs en pisciculture. Après incubation dans les installations recyclées INRA de Rennes, les œufs obtenus ont été introduits à Jouy au stade œillé.

\section{PROTOCOLE EXPÉRIMENTAL}

Les incubateurs sont constitués de paniers plastiques de $10 \mathrm{~cm} \times 10 \mathrm{~cm}$ au fond desquels est fixée une tôle plastique à perforations de $1,5 \mathrm{~mm}$. Le substrat rugeux a été obtenu en collant sur le fond des incubateurs un morceau de grillage plastique "Nortene" à mailles de $8 \times 5 \mathrm{~mm}$.

Les incubateurs sont placés par groupes de 4 dans des auges d'incubation de 7 litres, chaque auge correspondant à un croisement donné testé sur les deux substrats avec une répétition. Chaque incubateur contient 100 œufs embryonnés, à l'exception du $2^{\mathbf{e}}$ réplicat du génotype $E \times G$ qui n'en reçoit que 50. Pour tester d'éventuelles différences environnementales entre les auges, le lot issu des géniteurs prélevés sur l'Elorn est divisé en deux groupes (Elorn 1 et Elorn 2) occupant deux auges différentes. Chaque auge est alimentée par un débit d'environ $1 \mathrm{l} / \mathrm{min}$. à $10^{\circ} \mathrm{C}$ circulant de manière descendante dans les incubateurs.

\section{PARAMĖTRES EtTUdies}

La survie est estimée par le nombre d'alevins survivants 34 jours après l'éclosion (en \% du nombre d'œufs œillés mis en place).

Le poids frais est estimé à 34 et 41 jours par deux pesées de 25 individus dans chaque lot. L'écart entre les deux estimations fournit également une estimation de la variation individuelle intragroupe.

La teneur en eau des alevins est estimée à 41 jours sur deux échantillons de 15 individus par groupe. Les animaux sont congelés, puis placés dans une étuve à $80^{\circ} \mathrm{C}$ pendant 48 heures. Les mesures de poids sec sont effectuées sur une balance de précision ( $11 \mathrm{mg}$ ) et rapportées au poids initial.

\section{TRAITEMENT STATISTIQUE}

\subsection{Analyse des valeurs phénotypiques}

Une transformation angulaire $\left(p^{\prime}=\arcsin V \vec{p}\right)$ a été faite sur les pourcentages de survie pour permettre l'application des modèles d'analyse de variance (SNEDECOR et COCHRAN, 1957).

La distribution du poids frais avant le début de l'alimentation suivant approximativement une Ioi normale (CHEVASSUS, 1976), les modèles d'analyse de variance ont été appliqués sur les variables non transformées.

L'influence de la place de l'incubateur dans l'auge (amont ou aval) a été étudiée par le test des signes de Wilcoxon (SNEDECOR et COCHRAN, 1957).

L'absence de variations environnementales interauges a été testée en comparant les lots ELORN 1 et 2 par une analyse de variance à deux facteurs, l'un aléatoire (auge), l'autre fixé (substrat).

Les performances de croissance de la souche Elorn issue de géniteurs en provenance de la rivière ou de la pisciculture ont été comparées à l'aide du test de Student (SNEDECOR et COCHAAN, 1957).

Les effets de la souche et du type de substrat sur la survie, le poids frais, le poids sec et la teneur en eau ont été testés au sein du diallèle par une analyse de variance à deux facteurs fixés (souche, substrat) avec répétition: Yijk $=\mu+A i+B j+l i j+E i j k$ dans lequel $\mu, A i, B j$, lij et Eijk désignent respectivement la moyenne générale, l'effet génotype, l'effet substrat, l'interaction génotype-substrat et l'écart résiduel. 


\subsection{Analyse génétique}

Nous avons utilisé le modèle génétique suivant (GARDNER et EBERHART, 1986) : $\mathrm{Xij}=\mu+$ $1 / 2 \mathrm{gi}+1 / 2 \mathrm{gj}+\mathrm{mi}+\mathrm{Hij}$, dans lequel gi, gj, $\mathrm{mi}$ et $\mathrm{Hij}$ désignent respectivement la contribution génétique de la souche maternelle, celle de la souche paternelle, l'effet maternel et l'hétérosis entre les souches $i$ et $j$.

Pour un croisement diallèle, les simplifications suivantes peuvent être faites : $g i=-g j=g$, $\mathrm{mi}=-\mathbf{m j}=\mathbf{m}$ et $\mathrm{Hij}=\mathrm{H}$. Ce modèle a été appliqué, pour chaque type de substrat considéré séparément, à l'analyse des données relatives aux quatre paramètres étudiés (survie, poids frais. poids sec, teneur en eau). Pour tester la signification statistique de $\mathrm{g}$ et $\mathrm{H}$, nous avons effectué des analyses de variance à deux facteurs fixés (mâle, femelle). Le modèle statistique utilisé est identique à celui-ci qui est décrit dans le paragraphe 4.1.

\section{RÉSULTATS}

\section{ANALYSE statistiQue des VALEURS PHÉNOTYPIQUES}

Les valeurs moyennes observées sont présentées sur le Tableau I. Aucun effet de l'auge ou de la place dans l'auge (amont-aval) n'a pu être mis en évidence pour chacun des quatre caractères analysés.

L'utilisation du substrat rugueux se traduit par une amélioration significative de la survie à 34 jours, du poids vif à 34 et 41 jours, du poids sec à 41 jours (Tab. II, III, IV et V). Les gains de poids sont en moyenne de $+22 \%$ sur substrat lisse et $+35 \%$ sur substrat rugueux à 34 jours, de $+30 \%$ et $+41 \%$ à 41 jours (Tab. I). A 34 jours, les taux de survie s'établissent en moyenne à $87 \%$ sur substrat lisse et $94 \%$ sur substrat rugueux. Enfin, le poids sec est supérieur de $10 \%$ en moyenne sur substrat rugueux (Tab. I). La figure 1 illustre les gains de poids rapportés au poids des œufs œillés dans les différents groupes. Ces améliorations de performances concernent tous les lots étudiés, à l'exception du lot $\mathrm{E} \times \mathrm{E}$ dans le cas de la survie à 34 jours. La teneur en eau reste identique pour tous les lots quel que soit le type de substrat utilisé.

L'analyse des performances observées sur les lots du croisement diallèle fait apparaître un effet statistiquement significatif de la souche pour le poids vif et la survie à 34 jours ainsi que le poids sec à 41 jours (Tab. II, III, IV et V). Cet effet est particulièrement net dans le cas du poids vif qui est sensiblement amélioré par l'utilisation de géniteurs domestiques, les croisements $E \times G$ et $G \times E$ se situant entre les deux souches pures. Une interaction significative souchex substrat n'a pu être mise en évidence que pour la survie à 34 jours. II faut cependant remarquer que l'effet positif du substrat rugueux est proportionnellement plus important pour les lots $E \times E$ que pour les lots $G \times G$. Enfin, les poids vif et sec des individus issus de géniteurs prélevés dans l'Elorn sont significativement plus élevés que ceux des géniteurs Elorn élevés en pisciculture.

Tableau I: Survie et poids moyen des difterents groupes à 34 et 41 jours. Table I: $\quad$ Survival rate and mean weight of the different groups at 34 and 41 days.

\begin{tabular}{|c|c|c|c|c|c|c|c|}
\hline \multirow[b]{2}{*}{ GENOTYPE } & \multirow[b]{2}{*}{ SUBSTRAT } & \multirow{2}{*}{$\begin{array}{c}\text { POIDS MOYEA* } \\
\text { DES OEUPS } \\
\text { (E) }\end{array}$} & \multicolumn{2}{|c|}{34 JOURS } & \multicolumn{3}{|c|}{41 JoURS } \\
\hline & & & $\begin{array}{l}\text { SURVIE } \\
(z)\end{array}$ & $\begin{array}{c}\text { POIDS MOYEN } \\
\text { (ag) }\end{array}$ & $\begin{array}{l}\text { POIDS MOYEN } \\
\left(\begin{array}{l}(\mathrm{g}) \\
\end{array}\right.\end{array}$ & $\begin{array}{l}\text { TENEUR EN } \\
\text { EAU (Z) }\end{array}$ & $\begin{array}{c}\text { POIDS SEC } \\
\text { (ag) }\end{array}$ \\
\hline ELORN 1 & $\mathbf{L}$ & 93,8 & 90,0 & 101,3 & 104,1 & 83,0 & 17,7 \\
\hline (riviàre) & $\mathbf{R}$ & 98,9 & 98,0 & 117,9 & 118,7 & 83,8 & 19,2 \\
\hline ELORN 2 & $\mathbf{L}$ & 92,5 & 82,5 & 104,3 & 110,3 & 83,4 & 18,3 \\
\hline (riviàre) & $\mathbf{R}$ & 92,5 & 97,5 & 114,8 & 120,0 & 84,0 & 19,2 \\
\hline \multirow[t]{2}{*}{$E \times E$} & $\mathbf{L}$ & 61,1 & 88,0 & 71,7 & 80,7 & 84,6 & 12,4 \\
\hline & R & 60,9 & 87,5 & 87,8 & 89.5 & 85,3 & 13,2 \\
\hline \multirow[t]{2}{*}{$E \times G$} & $\mathbf{L}$ & 61,9 & 71,3 & 81,1 & 84,8 & 85,4 & 12,4 \\
\hline & $\mathbf{z}$ & 62,0 & 92,0 & 88,7 & 92,0 & 85.7 & 13,2 \\
\hline \multirow[t]{2}{*}{$G \times E$} & $\mathbf{L}$ & 70.9 & 95,5 & 92,2 & 98,1 & 84,5 & 15.2 \\
\hline & R & 69,8 & 96,0 & 99,6 & 105,6 & 84,5 & 16,3 \\
\hline \multirow[t]{2}{*}{$G \times G$} & $\mathbf{L}$ & 75,9 & 93,5 & 103,2 & 107,1 & 85,7 & 15,3 \\
\hline & R & 73,0 & 95,0 & 111,4 & 110,6 & 84,6 & 17.0 \\
\hline
\end{tabular}

\footnotetext{
*: le poids coyen de chaque lot experimental a 6 te esure et utilise pour calculer les potds a 34 et a 41 jours en $x$ du poids Initial. L: substrat lisse; R: substrat rugueux.
} 
Bull. Fr. Pêche Piscic. (1988) 311:126-133 - $129-$

Tableau II : Anabyse de variance de la survie a 34 jours.

Tablell: $\quad V$ ariance anabysis of the survival rate at 34 days.

\begin{tabular}{|c|c|c|c|c|}
\hline $\begin{array}{l}\text { SOURCE DE } \\
\text { VARIATION }\end{array}$ & DDL & $\begin{array}{l}\text { CARRE } \\
\text { MOYEN }\end{array}$ & $\mathbf{F}$ & $\begin{array}{l}\text { COMPOSANTES DE LA } \\
\text { VARIANCE EN } \Varangle\end{array}$ \\
\hline SOUCHE & 3 & 164,48 & $\star \star$ & 35,7 \\
\hline SUBSTRAT & 1 & 136,89 & $\star$ & 14,4 \\
\hline $\begin{array}{l}\text { INTERACTION } \\
\text { SOUCHE-SUBS? }\end{array}$ & 3 & 81,16 & $\star$ & 30,3 \\
\hline RES IDUELLE & 8 & 19,79 & & 19,6 \\
\hline
\end{tabular}

Tableau III : Analyse de variance du poids vif à 34 jours rapporté au poids des ceufs ceillés.

Table III: Variance anabysis of the wet weight at 34 days in percent of the eyed eggs weight.

\begin{tabular}{|c|c|c|c|c|}
\hline $\begin{array}{l}\text { SOURCE DE } \\
\text { VARIATION }\end{array}$ & DDL & $\begin{array}{l}\text { CARRE } \\
\text { MOYEN }\end{array}$ & $\mathbf{P}$ & $\begin{array}{l}\text { COYPOSANTES DE LA } \\
\text { VARIANCE EN } 7\end{array}$ \\
\hline SOUCHE & 3 & 259,11 & $\star \star$ & 11,6 \\
\hline SUBSTRAT & 1 & 2518,73 & $\star \star$ & 66,3 \\
\hline INTERACTION & 3 & 77,43 & NS & 3,7 \\
\hline \multicolumn{5}{|c|}{ SOUCHE-SUBSTRAT } \\
\hline RESIDUELLE & 8 & 42,86 & & 18,4 \\
\hline
\end{tabular}

**: signiflcatif a $1 x_{\text {; }}$ NS: non signiflcatif 
Tableau IV : Analyse de variance des poids vifs à 41 jours rapportés au poids des œuf́s œillés.

Table IV : Variance analysis of the wet weights at 41 days, in percent of the eyed eggs weight.

\begin{tabular}{lcccc}
\hline $\begin{array}{l}\text { SOURCE DE } \\
\text { VARIATION }\end{array}$ & DDL & $\begin{array}{c}\text { CARRE } \\
\text { MOYEN }\end{array}$ & F & $\begin{array}{l}\text { COMPOSANTES DE LA } \\
\text { VARIANCE EN } \%\end{array}$ \\
\hline SOUCHE & 3 & 98,13 & NS & 5,8 \\
SUBSTRAT & 1 & 1483,76 & $\star \star$ & 67,6 \\
$\begin{array}{l}\text { INTERACTION } \\
\text { SOUCHE-SUBSTRAT }\end{array}$ & 3 & 6,53 & NS & 0 \\
& & & & \\
RESIDUELLE & 8 & 35,55 & & 26,6 \\
\hline
\end{tabular}

**: significatif à $1 \%$; NS: non signifactif

Tableau V : Analyse de variance du poids sec à 41 jours.

Table V: $\quad$ ariance analysis of the dry weight at 41 days.

\begin{tabular}{lcccc}
\hline $\begin{array}{l}\text { SOURCE DE } \\
\text { VARIATION }\end{array}$ & DDL & $\begin{array}{c}\text { CARRE } \\
\text { MOYEN }\end{array}$ & F & $\begin{array}{l}\text { COMPOSANTES DE LA } \\
\text { VARIANCE EN } 7\end{array}$ \\
\hline SOUCHE & 3 & 29,56 & $\star \star$ & 71,3 \\
SUBSTRAT & 1 & 11,56 & $\star \star$ & 12,1 \\
$\begin{array}{l}\text { INTERACTION } \\
\text { SOUCHE-SUBSTRAT }\end{array}$ & 3 & 0,37 & NS & 0 \\
RESIDUELLE & 8 & 0,88 & & 16,3 \\
\hline
\end{tabular}

**: 81gniflcatif a $1 \pi$; NS: non significatif 
Tableau VI : Valeurs de paramètres génétiques $\mu, \mathrm{g}, \mathrm{m}$ et H du genotype Elorn bsu de géniteurs élevés en plsciculture. Pour le génotype La Gouarnais, les valeurs de $\mathrm{g}, \mathrm{m}$ et $\mathrm{H}$ sont obtenus par changement de signe.

Table VI: $\quad V$ alues of $\mu, g, m$ and $H$ for Elorn genotype issued from spawners reared at the hatchery. For La Gouarnais strain, the values of parameters $\mathrm{g}, \mathrm{m}$ and $\mathrm{H}$ are deduced by changing sign.

\begin{tabular}{|c|c|c|c|c|c|c|c|c|}
\hline \multirow[b]{2}{*}{ CARACTERE } & \multicolumn{4}{|c|}{ SUBSTRAT LISSE } & \multicolumn{4}{|c|}{ SUBSTRAT RUGUEUX } \\
\hline & $\mu$ & $\mathrm{g}$ & H & $m$ & $\mu$ & $\mathbf{g}$ & H & $m$ \\
\hline SURVIE & 72,47 & $+7,32$ & $-4,80$ & $-10,07$ & 73,20 & $-1,45$ & $+2,82$ & $-2,45$ \\
\hline POIDS $a 34 \mathrm{j} \ddagger$ & 129,00 & $-10,50$ & $-2,20$ & $-1,10$ & 148,30 & $-4,25$ & $-5,55^{\star}$ & $+0,05$ \\
\hline POIDS $a 41 \mathrm{f} \ddagger$ & 136,65 & $-3,95$ & $-1,15$ & $-0,60$ & 149,75 & $-1,25$ & $-0,05$ & $-1,60$ \\
\hline $\begin{array}{l}\text { POIDS SEC } \\
\text { a } 41 \mathrm{~J}\end{array}$ & 13,73 & $-0,54$ & $+0,44$ & $-1,03$ & 15,14 & $-0,36$ & $-0,42$ & $-1,60$ \\
\hline $\begin{array}{l}\text { TENEUR } \\
\text { en BAU }\end{array}$ & 85,17 & $-0,96$ & $-0,22$ & $+0,44$ & 84,96 & $-0,27$ & $-0,19$ & $+0,60$ \\
\hline
\end{tabular}

f: exprime en $z$ du polds soyen de 1 'oeuf au stade oetl1e; $*$ : significatif a $5 z$

Poids mogen (\% Poids oeuf́s oeillés)

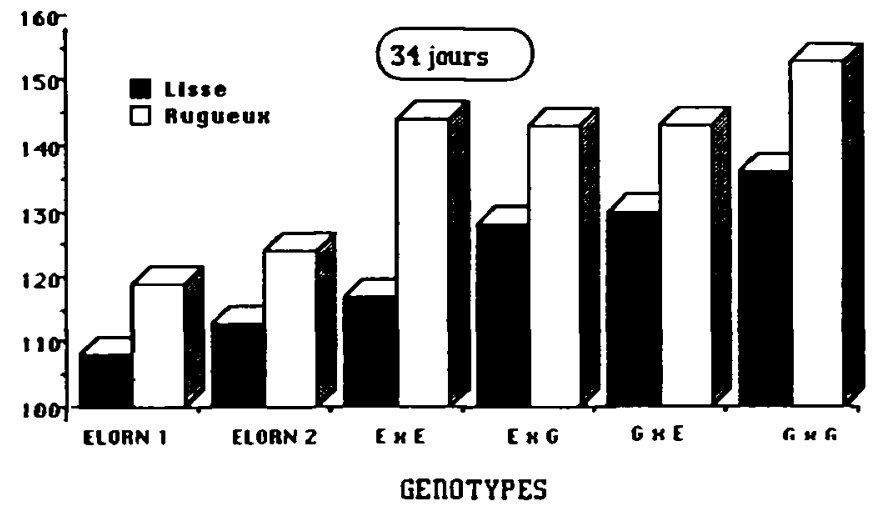

Poids mogen (\% Poids oeufs oeillés)

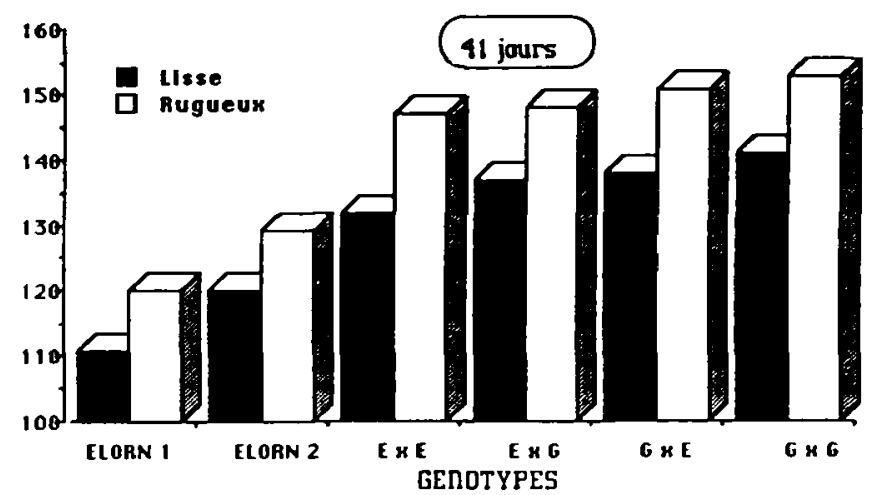

figure 1: Poids vif moyen des différents groupes en \% du poids des ceufs ceillés.

figure 1 : Mean wet weight in percent of eyed eggs weight. 


\section{ANALYSE GÉNEtiQue}

Aucun effet génétique significatif au seuil de $5 \%$ n'a pu ètre mis en évidence, à l'exception de l'hétérosis négative pour le poids à 34 jours (phénomène qui disparaît à 41 jours). Les effets maternels qui n'ont pas fait l'objet d'analyse statistique pourraient expliquer une part importante de l'effet souche observé.

\section{DISCUSSION ET CONCLUSION}

Cette ètude fait très clairement apparaître un effet bénéfique du substrat rugueux sur la survie et surtout la croissance au cours du développement précédant l'alimentation. Le gain de poids liè à l'utilisation du substrat rugueux s'établit à $10 \%$ en moyenne; il est cependant moins important que ceux qui ont été obtenus chez le saumon atlantique, 25 à $30 \%$ en moyenne (GAIGNON et PROUZET, 1982 ; ERIKSSON et WESTLUND, 1985).

L'augmentation de poids sec permet de préciser que l'effet positif du substrat rugueux ne correspond pas à une simple hydratation supplémentaire de l'œuf, mais se traduit par une meilleure efficacité dans l'utilisation des réserves vitellines de celui-ci. Des résultats similaires ont déjà été mentionnés dans le cas du saumon atlantique (HANSEN et TORRISEN, 1985).

L'effet souche mis en évidence dans cette étude peut résulter à la fois d'effets génétiques et d’effets maternels. L'analyse génétique que nous avons réalisée ne fait appparaître aucun effet génétique significatif. II faut toutefois remarquer qu'au sein du diallèle, pour une catégorie donnée de femelles, les alevins issus de mâles domestiques ont une croissance toujours égale ou supérieure à celle des descendants de mâles sauvages. Si nous considérons qu'il n'y a pas d'effet-auge, ces différences indiquent l'existence d'un effet-souche génétique. Le même phénomène avait déjà été observé pour la croissance et la survie dans un croisement diallèle impliquant les deux mêmes souches (GUYOMARD et CHEVASSUS, 1985). Cette concordance d'observations suggère fortement l'existence d'un effet génétique "souche" pour la croissance dans le cas considéré ici. L'existence d'effets maternels est également très probable. Ceux-ci peuvent expliquer en partie les différences de poids sec dans le cas du croisement diallèle ou les différences de poids vif entre les lots Elorn issus de géniteurs sauvages et de géniteurs domestiques. Chez la truite arc-en-ciel, l'effet de la taille de l'œuf sur celle de l'alevin a été fréquemment observé jusqu'à la fin du premier mois d'alimentation (GALL, 1974; SPRINGATE et BROMAGE, 1985) et tend à disparaître au-delà (SPRINGATE et BROMAGE, 1985).

L'amélioration des performances de survie et croissance d'une souche permet, d'une part, d'accroître sensiblement la quantité d'œufs produite grâce à l'augmentation du nombre et de la taille des femelles domestiques disponibles lors de la période de reproduction, d'autre part, de réduire toute évolution génétique sélective ou aléatoire lièe à de fortes mortalités. Dans cette perspective, l'effet positif du substrat rugueux mis en évidence est encourageant. Il doit être cependant confirmé au cours de la période de forte mortalité que constituent les trois mois suivant le début de l'alimentation dans le cas des souches sauvages (GUYOMARD et CHEVASSUS, 1985). Si une amélioration significative des performances était obtenue grâce au substrat rugueux au cours de cette période, cette méthode d'incubation pourrait être préconisée lors de la constitution de nouveaux stocks de pisciculture à partir de souches naturelles souvent difficiles à élever.

\section{REMERCIEMENTS}

Cette étude a été réalisée dans le cadre d'une convention CSP/INRA réf. 85329 "génétique des populations de truite commune". 


\section{BIBLIOGRAPHIE}

CHEVASSUS B., 1976. Variabilité et héritabilité des performances de croissance chez la truite arc-en-ciel (Salmo gairdneri R.) Ann. Bénét. Sél. Anim., 8, 273-283.

ERIKSSON C., WESTLUND G., 1983. The impact on survival and growth of Atlantic salmon (Salmo salar) and sea trout (Salmo trutta) by using incubators with artificial substrate. I. Hatching and first summer. Laksforkningsinst. Meddelande, 2, $16 \mathrm{p}$.

ERIKSSON C., WESTLUND G., 1985. The impact on survival and growth of atlantic salmon (Salmo salar) using incubators with artificial substrate. 1. Hatching and first summer. Laksforkningsinst. Meddelande, 2, $7 \mathrm{p}$.

GAIGNON J.L., PROUZET P., 1982. Élevage du saumon atlantique en écloserie: essai d'utilisation de substrats durant la résorption vitelline. Bull. Fr. Piscic., 287, 1-5.

GALL G.A.E., 1974. Influence of size of eggs and age of female on hatchability and growth in rainbow trout. Calif. Fish and Game, 1, 26-35.

GARDNER C.O., EBERHART S.A., 1966. Analysis and interpretation of the variety cross diallel and related populations. Biometrics, 22, 439-452.

GUYOMARD R., CHEVASSUS B., 1985. Recherches sur la génétique des populations de truite commune et de saumon atlantique. Compte-rendu de contrat INRA-CSP, 15 p.

HANSEN T., 1985. Artificial hatching substrate : effect on yolk absorbtion, mortality and growth during first feeding of sea trout (Salmo trutta) Aquaculture, 46, 275-285.

HANSEN T., TORRISSEN O., 1984. Artificial hatching substrate in mass rearing of larval atlantic salmon. ICES, Counc. Meet. F, 30, $11 \mathrm{p}$.

HANSEN T., MOLLER D., 1985. Yolk absorbtion, yolk sac constrictions, mortality and growth during first feeding of atlantic salmon (Salmo salar) incubated on astro-turf. Can. J. Fish. Aquat. Sci., 42, 1073-1078.

KRIEG F., GUYOMARD R., 1985. Population genetics of french brown trout (Salmo trutta L.) : large geographical differentiation of wild populations and high similarity of domesticated stocks. Génét. Sél. Evol., 17, 225-242.

LEON K.A., 1975. Improved growth and survival of juvenile atlantic salmon hatched in drums packed with a labyrinthine plastic substrate. Prog. Fish. Cult., 37, 158-163.

MAISSE G., PORCHER J.P., NIHOUARN A., CHEVASSUS B., 1983. Comparaison des performances en pisciculture d'un hybride intra-spécifique ( $O$ ' sauvage et $\$$ domestique) et de la souche domestique chez la truite commune (Salmo trutta). Essai préliminaire d'implantation en ruisseau. Bull. Fr. Piscic., 291, 167-181.

SNEDECOR G.W., COCHRAN W.G., 1957. Statistical methods. The lowa State University Press, AMES, IOWa, USA.

SPRINGATE J.R.C., BROMAGE N.R., 1985. Effects of egg size on early growth and survival in rainbow trout (Salmo gairdneri R.). Aquacultı $e, 47,163-172$. 